\title{
Energy Management of Hybrid Power System in UUV Based on Pontryagin's Minimum Principle
}

\author{
Huizhong Gao, Junguang Wang, Jun Lu *, Xiao Zong \\ Xi'an precision mechanical research institute, 710077, Xi'an PR China
}

\begin{abstract}
Hybrid power system which consists of a proton exchange membrane fuel cell and a battery has great perspective for Unmanned Underwater Vehicles (UUVs). As fuel cell's working point has a direct relationship with its efficiency and lifetime, a key concern of the hybrid power system is the power distribution between the two parts. Aiming at minimizing the equivalent hydrogen cost, this work presents an optimal real-time energy management strategy based on the Pontryagin's minimum principle. Compared with some other methods, this method possesses shorter calculation time and smaller storing space, which is helpful for online optimization in actual applications. In this research, a typical UUV working profile is tested with different initial co-state values to evaluate the proposed method, and the results indicate good robustness and effectiveness.
\end{abstract}

\section{Introduction}

In the past decades, many countries have put a lot of efforts to evolve underwater unmanned vehicles (UUVs) from lab research into commercial, military and scientific applications[1]. With the outstanding advances, UUVs have become the key role in ocean development. Generally, UUVs are characterized with extended range, long endurance, and low noise. Current fleet of UUVs is usually battery powered [2][3] as batteries are a simple solution. This factor combined with a battery's silence and ability to turn on and off instantly make it very attractive for UUVs. However, the potential for battery to enhance its energy density is quite limited. As such, battery technology cannot catch up with the UUV development with extended range and longer endurance.

A hybrid power system in UUVs often takes fuel cell as the main power source and battery as the auxiliary power source. In this condition, the energy management strategy (EMS) is required to split the power between the two sources [4]. For better performance, EMS should take the load demand and resource constraints into consideration. Ideally, by controlling the DC/DC converter's output current, EMS ensures the hybrid power system works at high efficiency. Previously, various EMS, such as rule-based strategies [5], instantaneous/global optimized strategies [6], intelligent algorithms [7][8] are studied. Pontryagin's minimum principle (PMP) is an optimal control technique firstly proposed by Pontryagin, which transforms the global optimization problem described by DP to an instantaneous Hamiltonian optimization problem. It doesn't need the whole cycle as prior information and can find the quasi optimal solution, which is proper to solve UUVs' online energy management problem.

In this paper, PMP-based EMS is studied for UUV application. Section 2 describes the structure of the fuel cell hybrid power system. Section 3 makes an introduction of the PMP theory, and raises the energy management problem. Then, in section 4, PMP strategy is used to achieve the solution, and simulation test is conducted to verify the method. Eventually, the conclusion is given in section 5 .

\section{The hybrid powertrain}

There are several powertrain topologies as candidates for hybrid power system. As the fuel cell is efficient and the battery has good dynamics, in this paper, a unidirectional DC is equipped on the fuel cell side and the battery is connected on bus directly. The whole powertrain is illustrated in Fig.1.

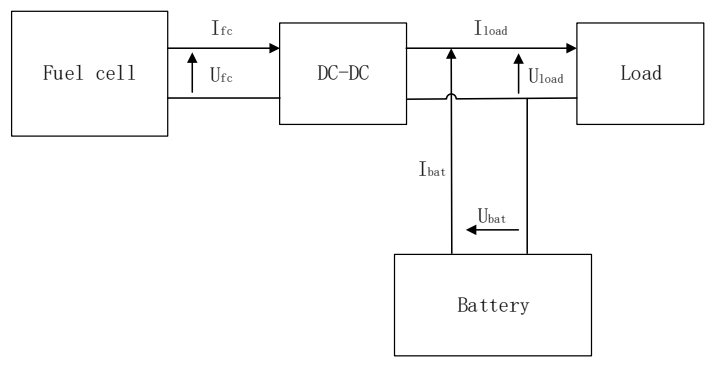

Fig.1. The topology of the FC hybrid power system

\subsection{Fuel cell model}

\footnotetext{
* Corresponding author: lujun_705@163.com
} 
Different from the hydrogen-air FC in buses, the hydrogen-oxygen FC in UUVs take the pure oxygen as oxidant. Therefore, the partial pressure in cathode is relevantly different. In [9], the author makes a comprehensive study on FC model.

According the electric chemical theory of $\mathrm{FC}[10]$, a single cell output voltage can be defined as a combination of the thermodynamic potential $E_{\text {nernst }}$, the activation overvoltage $V_{a c t}$ and the ohmic overvoltage $V_{\text {ohm }}$, coefficients of activation over voltage $\xi_{i}$.

$$
V_{\text {cell }}=E_{\text {energy }}-V_{\text {act }}-V_{\text {ohm }}
$$

where

$$
\begin{gathered}
E_{\text {nernst }}=\left(\begin{array}{c}
E_{0}-0.85 * 10^{-3}\left(T_{\text {body }}-298.15\right) \\
+\frac{R T_{\text {body }}}{2 F} \ln \left[p_{\mathrm{H}_{2}} *\left(p_{\mathrm{O}_{2}}\right)^{0.5}\right]
\end{array}\right) \\
V_{\text {act }}=-\left(\begin{array}{c}
\xi_{1}+\xi_{2} T_{\text {body }}+\xi_{3} T_{\text {body }} \ln C_{O_{2}} \\
+\xi_{4} T_{\text {body }} \ln I
\end{array}\right) \\
\xi_{2}=\left(\begin{array}{c}
0.00286+0.0002 \ln A_{c e l l} \\
+4.3 * 10^{-5} \ln C_{H_{2}}
\end{array}\right) \\
C_{H_{2}}=9.174 * 10^{-7} p_{H_{2}} \exp \left(\frac{-77}{T_{b o d y}}\right) \\
C_{O_{2}}=1.97 * 10^{-7} p_{O_{2}} \exp \left(\frac{498}{T_{b o d y}}\right) \\
r_{\text {mem }}=\frac{181.6\left[1+0.03 J+0.062\left(T_{\text {body }} / 303\right)^{2} J^{2.5}\right]}{[\gamma-0.634-3 J] \exp \left[4.18\left(\left(T_{\text {body }}-303\right) / T_{\text {body }}\right)\right]} \\
R_{\text {int }}=\frac{r_{\text {mem }} * R_{\text {mem }}}{A_{\text {cell }}} \\
V_{\text {oht }}
\end{gathered}
$$

Additionally, the saturated vapour pressure $p_{\mathrm{H}_{2} \mathrm{O}}^{\text {sat }}$ can be calculated by the operating temperature, and the effective partial pressure $p_{\mathrm{H}_{2}}$ and $p_{\mathrm{H}_{2}}$ are described as follows:

$$
\begin{gathered}
\log \left(p_{\mathrm{H}_{2} \mathrm{O}}^{\text {sat }}\right)=\left(\begin{array}{c}
2.95^{*} 10^{-2}\left(T_{\text {body }}-273.15\right)- \\
9.18^{*} 10^{-5}\left(T_{\text {body }}-273.15\right)^{2}+ \\
1.44 * 10^{-7}\left(T_{\text {body }}-273.15\right)^{3}-2.18
\end{array}\right) \\
p_{\mathrm{H}_{2}}=0.5 p_{\mathrm{H}_{2} \mathrm{O}}^{\text {sat }}\left[\frac{1}{X_{\mathrm{H}_{2} \mathrm{O}}^{\text {channel }} \exp \left(\frac{1.653 J}{T_{\text {body }}^{1.334}}\right)}-1\right]
\end{gathered}
$$

$$
p_{\mathrm{O}_{2}}=p_{\mathrm{H}_{2} \mathrm{O}}^{\text {sat }}\left[\frac{1}{X_{\mathrm{H}_{2} \mathrm{O}}^{\text {chanel }} \exp \left(\frac{4.192 J}{T_{\text {body }}^{1.334}}\right)}-1\right]
$$

Based on the analysis all above, the FC stack output voltage is calculated by

$$
V_{\text {stack }}=N_{\text {cell }} * V_{\text {cell }}
$$

\subsection{Battery model}

The Rint model is widely used to describe the performance of the battery as showed in Fig.2, in which the battery is transferred into a voltage source and a resistor in series.

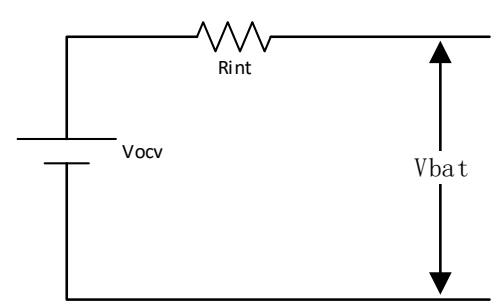

Fig.2. The Rint model

Usually the variation of the internal resistance of charge and discharge of battery has been related to the state of charge (SOC). Generally the current and the voltage can be expressed as below, when the battery power is $P$,

$$
\begin{gathered}
I_{b a t}=\frac{V_{o c v}-\sqrt{V_{o c v}^{2}-4 R_{\mathrm{int}} P}}{2 R_{\mathrm{int}}} \\
V_{b a t}=V_{o c v}-R_{\mathrm{int}} I_{b a t}
\end{gathered}
$$

where

$$
\begin{gathered}
V_{\text {ocv }}=\left\{\begin{array}{l}
f_{1}(S O C), \text { ch } \arg e \\
f_{2}(S O C), \text { discharge }
\end{array}\right. \\
R_{\text {int }}=\left\{\begin{array}{l}
f_{3}(S O C), \text { ch } \arg e \\
f_{4}(S O C), \text { discharge }
\end{array}\right.
\end{gathered}
$$

Furthermore, the battery SOC can be achieved by

$$
S O C=S O C_{0}-\int_{0}^{t} \frac{I_{b a t}}{Q_{b a t}} d t
$$

where $Q_{b a t}$ is the capacity of the battery.

\section{Energy management of hybrid power system based on PMP algorithm}

\subsection{Pontryagin's minimum principle}


The Pontryagin's minimum principle (PMP) is a very valid approach in optimal control theory. Although not sufficient, every solution that satisfies the necessary conditions is called an extremal solution. Obviously, if the optimal solution exists, it must be one of the extremal solutions.

Generally, the optimal control problem is defined to seek an accessible control variable $u^{*}$ that lead the system $x(t)=f[x(t), u(t), t]$ to follow a certain trajectory $x^{*}$ that minimizes the performance index $\mathrm{J}$.

$$
J(u)=\left(\begin{array}{l}
\Phi\left[x\left(t_{f}\right), t_{f}\right]+ \\
\int_{t_{0}}^{t_{f}} L[x(t), u(t), t] d t
\end{array}\right)
$$

On one hand, $L[x(t), u(t), t]$ is the instantaneous cost function and its integration is integral cost, which decide the performance of $x(t)$ and $u(t)$. On the other hand, $\Phi\left[x\left(t_{f}\right), t_{f}\right]$ is the terminal cost which can evaluate the terminal state of the system. In PMP theory, furthermore, a Hamilton function is given,

$$
H[x(t), u(t), \lambda(t), t]=\left(\begin{array}{c}
L[x(t), u(t), t]+ \\
\lambda^{T}(t) f[x(t), u(t), t]
\end{array}\right)
$$

where $\lambda(t)$ is a Lagrange multiplier called co-state. Then, the following relations are established:

$$
\begin{gathered}
\dot{x}^{*}(t)=\frac{\partial H}{\partial \lambda(t)}\left[x^{*}(t), u^{*}(t), \lambda(t), t\right] \\
\dot{\lambda}(t)=-\frac{\partial H}{\partial x^{*}(t)}\left[x^{*}(t), u^{*}(t), \lambda(t), t\right] \\
H\left[x^{*}(t), u^{*}(t), \lambda(t), t\right] \leq H[x(t), u(t), \lambda(t), t]
\end{gathered}
$$

\subsection{Energy management based on PMP}

UUV works in the underwater where the fuel/oxidizer cannot be acquired from the outside environment. It is critical for EMS to take optimal utilization of the onboard energy reserves. Because the battery chargingdischarging process may cause energy loss, the battery should be charged by fuel cell as less as possible to reduce the energy loss. Therefore, the objective function of the UUV's EMS is defined as

$$
J=\min \sum\left(\dot{m}_{b a t}+\dot{m}_{f c}\right)
$$

Considering the system features and the analysis above, the state equation is described as follow,

$$
\dot{x}=S \dot{O} C=-\frac{I_{b a t}}{Q_{b a t}}
$$

$$
I_{b a t}=\frac{V_{o c}-\sqrt{V_{o c}^{2}-4 R_{\mathrm{int}} P_{b a t}}}{2 R_{\mathrm{int}}}
$$

where $Q_{b a t}$ is the battery capacity, $I_{b a t}$ is the battery current and $V_{o c}$ is the open circuit voltage of the battery.

The equivalent hydrogen consumption of battery $\dot{m}_{b a t}$ is a function of battery power $P_{b a t}$

$$
\dot{m}_{b a t}=P_{b a t}\left(\eta_{c h g} \eta_{d i s}\right)^{-\operatorname{sgn}\left(P_{b a t}\right)}
$$

To solve the allocation problem of hybrid power system, according to the PMP, the Hamilton function is defined below,

$$
\begin{gathered}
H=\dot{m}_{b a t}+\dot{m}_{f c}-\lambda * \frac{I_{b a t}}{Q_{b a t}} \\
\operatorname{SOC}(t)=\frac{\partial H}{\partial \lambda}=-\frac{V_{o c}(S O C)-\sqrt{V_{o c}^{2}(S O C)-4 R_{\text {int }} P_{b a t}}}{2 R_{\text {int }} Q_{b a t}} \\
\dot{\lambda}(t)=-\frac{\partial H}{\partial S O C}=\frac{\lambda(t)}{Q_{b a t}}\left(\begin{array}{l}
\frac{\partial I_{b a t}}{\partial V_{o c}}+\frac{\partial V_{o c}}{\partial S O C} \\
+\frac{\partial I_{b a t}}{\partial R_{\text {int }}} * \frac{\partial R_{\text {int }}}{\partial S O C}
\end{array}\right)
\end{gathered}
$$

Additionally,

$$
\dot{\lambda}(t)=-\frac{\lambda(t)}{Q_{b a t}} * \frac{I_{b a t}}{\sqrt{V_{o c}^{2}(S O C)-4 R_{\text {int }} P_{b a t}}} * \frac{\partial V_{o c}}{\partial S O C}
$$

At any instant, there will be an optimal control variable $P_{d c}^{*}$ makes $\mathrm{H}$ reaches its minimum value

$$
P_{d c}^{*}=\arg \min H
$$

Based on this, the corresponding battery power $P_{b a t}^{*}$ can be easily calculated with corresponding constraints

$$
P_{b a t}^{*}=P_{r e q}-P_{d c}^{*}, P_{b a t} \in\left[P_{b a t, \text { max }}, P_{b a t, \text { min }}\right]
$$

\subsection{Energy management problem solution}

Commonly, the PMP-based optimal problem can be converted into a two-point boundary value problem, and utilize a numerical method to solve the optimization of the Hamiltonian function. Shooting method is a widely used numerical approach in differential equation solving, whose cardinal process is to seek out optimal initial costate $\lambda_{0}^{*}$ iteratively. In other words, only the proper selected $\lambda_{0}^{*}$ can lead to a satisfied shooting process. By using the Secant method, the initial co-state is modified at each shooting process until the condition $\operatorname{SOC}\left(t_{f}\right)=\mathrm{SOC}_{\mathrm{L}}$ is fulfilled, whose form is 


$$
\left\{\begin{array}{c}
\lambda_{1}=\lambda_{0}, i=1 \\
\lambda_{2}=\lambda_{1}+\delta, i=2 \\
\lambda_{i}=\lambda_{i-1}-\left(\lambda_{i-1}-\lambda_{i-2}\right) * \frac{S O C_{i-1, t_{f}}-S O C_{t_{f}}}{S O C_{i-1, t_{f}}-S O C_{i-2, t_{f}}}, i=3,4, \ldots
\end{array}\right.
$$

where $\lambda_{i}$ is the initial co-state value in $\mathrm{i}$-th shooting process, $S O C_{i, t_{f}}$ is the end SOC in i-th shooting process.

During the solving procedure, the SOC and $\lambda$ are changed along with their derivatives in the form of

$$
\begin{gathered}
S O C_{i}^{k+1}=S O C_{i}^{k}+S \dot{O} C^{*} \Delta t \\
\lambda_{i}^{k+1}=\lambda_{i}^{k}+\dot{\lambda} * \Delta t
\end{gathered}
$$

Eventually, an intelligent iterative strategy based on instantaneous data by trial and error over a several simulations and contrast is able to be implemented.

\section{Simulation and discussion}

The hybrid UUV usually works in periodic power cycle and supplies enough energy and power in its mission. In order to verify the effect of PMP-based energy management strategy, a typical power profile of smallsized UUV is simulated in this section, as depicted in Fig.3. The specifications of the fuel cell/battery hybrid power system and the PMP parameters are showed in table 1 .

Table 1. System parameters

\begin{tabular}{ll}
\hline Parameter item & value \\
\hline Fuel cell max power & $5000 \mathrm{~W}$ \\
Battery max power & $3000 \mathrm{~W}$ \\
Capacity & $9.6 \mathrm{Ah}$ \\
SOC upper level & $90 \%$ \\
SOC lower level & $25 \%$ \\
$\Delta \mathrm{P}$ & $1 \mathrm{~W}$ \\
$\Delta \mathrm{t}$ & $1 \mathrm{~s}$ \\
$\lambda_{0}$ & $-10 /-15 /-20$ \\
$\delta$ & -4 \\
$\mathrm{E}$ & 0.001 \\
\hline
\end{tabular}

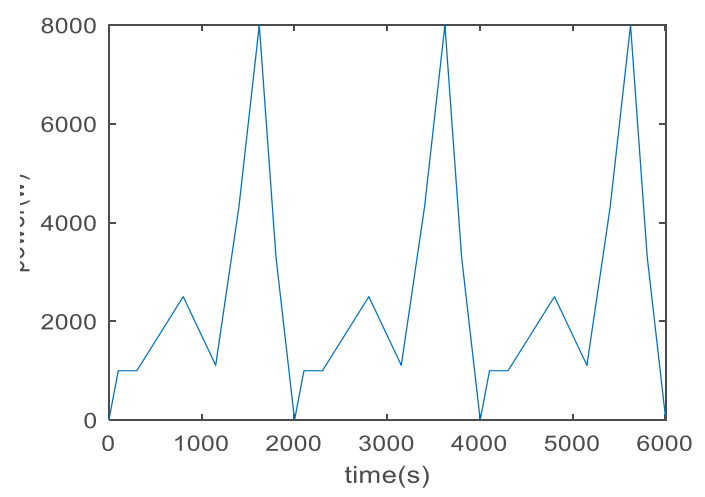

Fig.3. Power profile

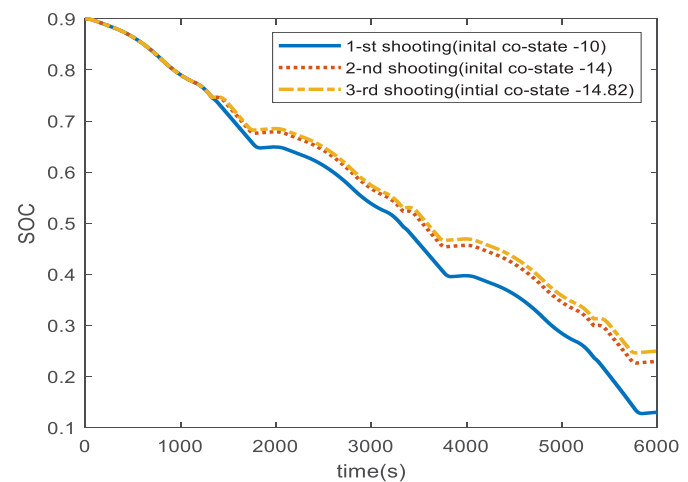

Fig.4. SOC trajectory of $\lambda_{0}=-10$

Fig. 3 shows the power profile, meanwhile, the optimal trajectories of SOC are illustrated in Fig. 4-6.

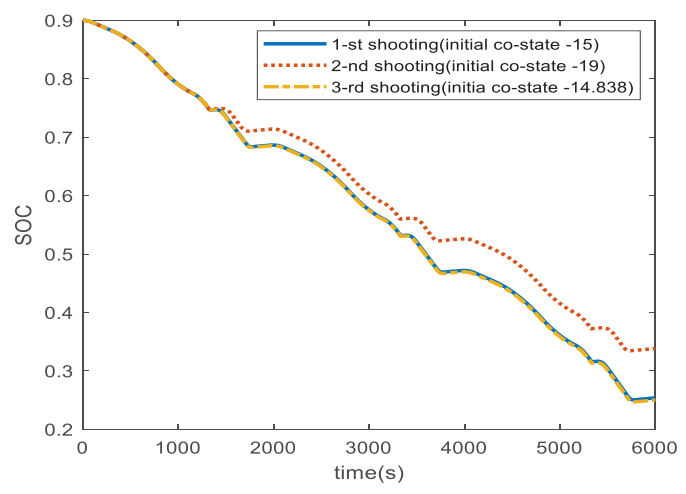

Fig.5. SOC trajectories of $\lambda_{0}=-15$

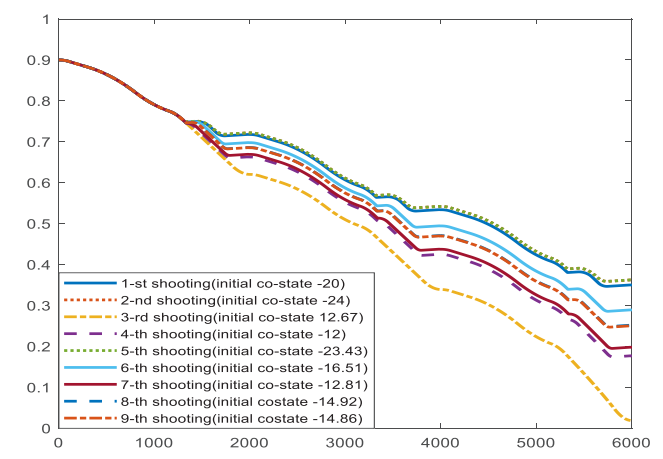

Fig.6. SOC trajectories of $\lambda_{0}=-20$

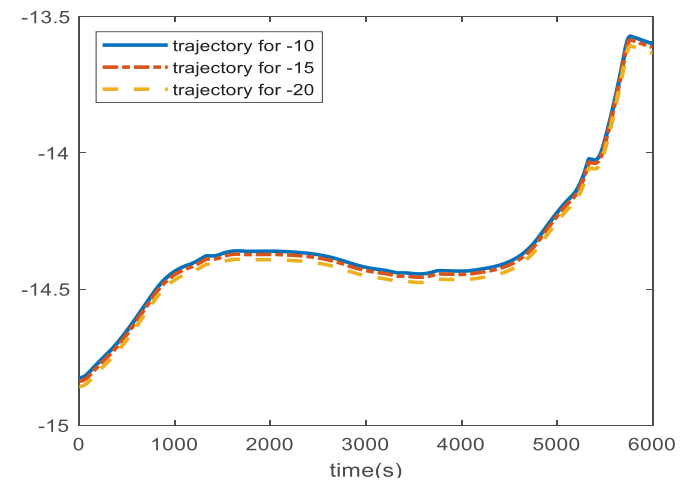

Fig.7. Optimal trajectories 
In the presented algorithm, by precisely regulating the power distribution during the mission process, the battery SOC is well decline from the initial value to a permitted minimum. The results in Fig.4-Fig.6 indicate that, although the shooting sequences start from different initial co-state value, they can eventually reach almost the same optimal co-state value $\left(-14.825\right.$ for $\lambda_{0}=-10$,14.837 for $\lambda_{0}=-15$, and -14.858 for $\lambda_{0}=-20$ ), specifically, the optimal co-state in the case of 3 distinct initial value that lead SOC change from $90 \%$ to $25 \%$ is also shown in Fig.7. Consequently, the optimal energy split path can be achieved through the optimal co-state and the trajectory.

\section{Conclusion}

This paper studies PMP and applies it to EMS of hybrid power system in UUVs. In the simulation, UUV's power profile is utilized to verify the optimal energy management strategy and a set of conclusions can be summarized as follow:

- The numerical solution of the PMP approach is achieved by using shooting method, which converts a constrained optimization problem into a two-point boundary value problem. In the calculation process, the Secant method can rapid tune the co-state $\lambda$ to the optimal values, which satisfy both the power and SOC demand and minimize the consumption.

- The presented algorithm is examined by a simulated power profile with 3 different initial co-state values. The results indicate that the identical problem with different initial values has totally distinct calculation process, but can eventually reach the similar optimal solution. Therefore, the PMP-based EMS is robust and can offer an effective online solution for the UUVs' hybrid power system.

\section{References}

1. Vukic.http://www.aig.si/13/clanki/vabljena_predava nja/U3_Vukic.pdf. (2013).

2. Alam, K., Ray, T., Anavatti, S.G. Ocean. 88(2014), 627-630.

3. Alaaeldeen M.E.Ahmed, Duan Wen-yang. Journal of ship mechanics, 2016, 20,6: 768-787(2016).

4. A. Tahri, H. El Fadil, F.Z. Belhaj, et al. Manegement of fuel cell power and supercapacitor state-of-charge for electric vehicles.Electric Power Systems Research, 160:89-98(2018).

5. Schouten NJ, Salman MA, Kheir NA. IEEE T Contr Syst $T, \mathbf{1 0 , 3 : 4 6 0 - 4 6 8 ( 2 0 0 2 ) . ~}$

6. Xu L, Li J, Hua J, Li X, Ouyang M. Int J Hydrogen Energy, 34:7323-33(2009).

7. Xiaohui Zhang, Li Liu, Yueling Dai, et al. International journal of hydrogen energy, 43:1009410103(2018).
8. Hou C , Ouyang M , Xu L , et al. Applied Energy, 115:174-189(2014).

9. Chien-Hsing Lee, Jian-Ting Yang. Journal of Power Sources, 196: 3810-3823(2011).

10. Wang $\mathrm{C}$, Nehrir $\mathrm{M} \mathrm{H}$, Shaw $\mathrm{S} \mathrm{R}$. IEEE Transactions on Energy Conversion, 20,2:442451(2005). 